\title{
Evaluation of perilipin 2 and melanocortin 5 receptor serum levels with sebogenesis in acne vulgaris patients
}

\author{
Özge Mine Örenay ${ }^{凶}$, Evren Sarıfakıoğlu², Ayla Gülekon³
}

${ }^{1}$ Department of Dermatology, Ankara Training and Research Hospital, Ministry of Health, Ankara, Turkey. ${ }^{2}$ Department of Dermatology, Private Gestalt Clinic, Ankara, Turkey. ${ }^{3}$ Department of Dermatology, Faculty of Medicine, Gazi University, Ankara, Turkey.

\begin{abstract}
Introduction: Acne vulgaris is a multifactorial disease. One of the main factors that plays a role in acne pathogenesis is an increase in sebum secretion. For sebum secretion, sebocyte differentiation followed by sebogenesis is essential. Sebocyte differentiation and proliferation, and sebum synthesis are controlled by complex pathways. Studies have shown that perilipin 2 and melanocortin 5 receptors play a role in sebogenesis. This study sought to determine whether levels of perilipin 2 and melanocortin 5 receptors have an impact on the development of acne vulgaris.

Methods: A total of 65 patients diagnosed with acne and 43 healthy control subjects were included in the study. Perilipin 2 and melanocortin 5 receptor levels were analyzed using the enzyme-linked immunosorbent assay (ELISA) technique.

Results: No significant differences were observed between the acne group and the control group in serum perilipin $2(p=0.594)$ and melanocortin 5 receptor $(p=0.213)$ levels. In the moderate acne group, perilipin 2 and melanocortin 5 receptor levels were significantly higher than in the mild acne group $(p=0.0014, p=0.003)$. The levels in the severe acne group were not higher compared to the moderate and mild acne groups.

Conclusions: This study failed to detect any association between acne pathogenesis and perilipin 2 and melanocortin 5 receptor serum levels. However, these proteins may have an influence on acne severity.
\end{abstract}

Keywords: acne vulgaris, melanocortin 5 receptor, perilipin 2, sebogenesis

Received: 20 June 2020 | Returned for modification: 27 September 2020 | Accepted: 21 October 2020

\section{Introduction}

Acne pathogenesis is multifactorial, and one of the main factors that leads to acne is increased sebum secretion (1). Acne treatment options depend on variable factors such as the type and severity of the acne, the patient's age, skin type, comorbid disease history, previous medication history, and lifestyle (2). Topical therapies (such as benzoyl peroxide, retinoids, antibiotics, and azelaic acid), oral antibiotics, isotretinoin, hormonal agents, and physical modalities can be used to treat acne vulgaris. Oral isotretinoin is an isomer of retinoid acid, primally used to treat severe acne vulgaris and also to treat moderate acne that is treatment-resistant or acne that relapses quickly after discontinuation of other therapies. Isotretinoin affects sebocytes, which leads to sebum suppression $(2,3)$.

Sebocytes form sebaceous glands, store lipid droplets (LDs) in their cytoplasm, and synthesize sebum. Proliferation, differentiation of sebocytes, and sebum synthesis are controlled by complex pathways (4). LDs, which provide lipid storage, are found in most cells, especially in adipose tissue $(5,6)$. During differentiation, sebocytes accumulate LDs in their cytoplasm. The main feature of sebocyte differentiation is the accumulation of lipids in LDs. Therefore, proteins on the surface of the LDs are thought to play a role in sebocyte differentiation (7). There are many proteins on the surface of LDs. The perilipin family is one of the important protein families that exist on the LD surface. The perilipin family contains five known proteins: perilipin (PLIN) 1 to 5. PLINs have different impacts in different tissues (6). Studies have revealed various proteins, including PLIN, in mice and human sebocytes (8-11). In a study to detect LD-related proteins in SZ95 (human se- bocyte culture model), 54 proteins were detected, including PLIN2 and PLIN3 (9).

Melanocortins synthesized by the proopiomelanocortin (POMC) gene include adrenocorticotrophin hormone (ACTH) and melanocyte-stimulating hormone ( $\mathrm{MSH} a, \beta, y$ ). In the skin, melanocortins play a role in regulating pigmentation, immune response, and exocrine gland secretion $(12,13)$. Melanocortins act by binding to their specific receptors. There are five melanocortin receptors (MC-R): MC1- $\mathrm{R}$ to $\mathrm{MC}_{5}-\mathrm{R}$ (11). $\mathrm{MC}_{5}$-Rs have been detected in mast cells and sebocytes $(14,15)$. In human sebaceous glands, expression of both MC1-R and MC5-R was detected $(16,17)$. Apart from androgens, other hormones such as POMC also have effects on sebaceous gland function. The effects of a-MSH on sebaceous glands were shown in a study by Thody et al. (18). Ablation of the pituitary gland of rats led to decreased lipid production in the sebaceous gland. Furthermore, injection of testosterone and a-MSH into these rats increased sebum production. These findings indicate that a-MSH is a sebotropic factor $(12,18)$. Another study showed that mice with POMC deficiency had a similar phenotype to $\mathrm{MC}_{5}$-R-deficient mice (indicating a possible role of $\mathrm{MSH}$ ). This finding supports the hypothesis that POMCs act through MC5-R (16). In a study by Chen et al. (19), a decrease in lipid secretion was observed in the skins of mice lacking $\mathrm{MC}_{5}$ - $\mathrm{R}$. In an in vitro study, increased levels of $\mathrm{MC}_{5}$ - $\mathrm{R}$ expression were detected in differentiated sebocytes induced by a-MSH (16). A different study showed MC5-R expression only in differentiated sebocytes (12).

Several studies showed that PLIN2 and MC5-R play a role in sebogenesis. In light of these findings, we examined the correlation between acne pathogenesis and acne severity with PLIN2 and MC5-R levels. 


\section{Materials and methods}

\section{Study population}

This study was conducted in patients that visited the Turgut Özal University Dermatology Outpatient Clinic between May 2014 and May 2015. A total of 65 patients ( 42 female and 23 male), ages 15 to 25 and diagnosed with acne vulgaris, were included in the study. A total of 46 ( 28 female and 18 male) healthy volunteers in the same age range as the acne group were included in the control group.

Pregnant or lactating women and patients with chronic liver disease, chronic kidney disease, hyperlipidemia, diabetes mellitus, body mass index (BMI) > 30, a diagnosis of polycystic ovarian syndrome (PCOS), and hirsutism were excluded from the study. Patients that had used systemic or topical acne treatment within a 1-month period were also excluded from the study.

\section{Acne severity measurement}

Patients diagnosed with acne vulgaris were divided into three groups: mild (1-18 points), moderate (19-30 points), and severe acne ( $\geq 31$ points) based on a global acne grading system (according to the lesion in the region; no lesion $=0$ points, comedone $=1$ point, papule $=2$, pustule $=3$, nodule $=4$. The region factor was calculated as forehead $=2$ points, right cheek $=2$, left cheek $=2$, nose $=1$, chin $=1$, and chest $/$ back $=3$. The local region score was the region factor $\times$ lesion score. The total acne score was calculated by summing the scores of all regions) developed by Doshi et al (20).

\section{Sample collection and measurement}

In both groups, 8- to 12-hour fasting venous blood samples were taken for PLIN2 and MC5-R measurement. After resting at room temperature for 10 minutes, the blood samples were centrifuged at 3,000 rpm for 20 minutes. The samples were analyzed with a double-antibody sandwich enzyme-linked immunosorbent assay (ELISA) technique. The ELISA kits (Sunred Biological Technology Co.) covered monoclonal antibodies specific to antigens (PLIN2 and $\left.\mathrm{MC}_{5}-\mathrm{R}\right)$. Forty $\mu \mathrm{l}$ of serum was added to the plaques containing monoclonal antibody. Subsequently, $10 \mu \mathrm{l}$ of biotin-labeled antibody and $50 \mu \mathrm{l}$ of streptavidin-HRP were added. The samples were incubated at $37^{\circ} \mathrm{C}$ for 60 minutes. The washing procedure was performed for eliminating unbound antibodies. After adding $50 \mu \mathrm{l}$ of solutions A and B, the samples turned blue. The samples were incubated at $37^{\circ} \mathrm{C}$ for 10 minutes, and then a stop solution was added to stop the enzymatic reaction. Finally, the samples were evaluated with an automatic ELISA reader.

\section{Statistical analysis}

The results were evaluated as mean \pm standard deviation $(S D)$. The normality of the numerical variables was analyzed using the Shapiro-Wilks test and the homogeneity of the variances with Levene's test. To investigate the difference between the two groups' averages in terms of numerical variables, in parametric conditions a $t$-test was used, and in non-parametric conditions the Mann-Whitney $U$ was used. For comparison of more than two groups, ANOVA was used in parametric and the Kruskal-Wallis test in non-parametric conditions. For multiple comparisons, Tamhane's test was used. A $p$-value less than 0.05 was accepted as significant.

\section{Results}

The study included 42 females (65\%) and 23 males (35\%), or a total of 65 people diagnosed with acne vulgaris. The control group included 28 females (61\%) and 18 males (39\%), or a total 43 people with no chronic disease.

In the acne group, $11(17 \%)$ had mild acne, 31 (48\%) had moderate acne, and $23(35 \%)$ had severe acne. Nine $(21 \%)$ females and $2(9 \%)$ males had mild acne. Twenty-two (52\%) females and $9(39 \%)$ males had moderate acne. Eleven (26\%) females and 12 (52\%) males had severe acne.

There was no significant difference between the acne and control groups' age, height, weight, BMI, and PLIN2 and MC 5 -R levels ( $p>0.05$; Table 1).

Table 1 | Physical features, and perilipin 2 and melanocortin 5 receptor levels in the acne and control groups.

\begin{tabular}{lccc}
\hline Variable & $\begin{array}{c}\text { Acne group } \\
\text { mean } \pm \text { SD }\end{array}$ & $\begin{array}{c}\text { Control group } \\
\text { mean } \pm \text { SD }\end{array}$ & $\boldsymbol{p}$ \\
\hline Age & $19.60 \pm 4.10$ & $20.74 \pm 2.50$ & 0.073 \\
Height & $169.09 \pm 9.53$ & $168.15 \pm 9.94$ & 0.616 \\
Weight & $62.87 \pm 12.70$ & $64.80 \pm 13.90$ & 0.449 \\
BMl & $21.85 \pm 3.28$ & $22.75 \pm 3.44$ & 0.169 \\
PLIN2 $(\mathrm{ng} / \mathrm{ml})$ & $27.31 \pm 21.33$ & $27.23 \pm 20.21$ & 0.594 \\
MC5-R $(\mathrm{ng} / \mathrm{ml})$ & $14.52 \pm 10.48$ & $16.39 \pm 10.32$ & 0.213 \\
\hline
\end{tabular}

$\mathrm{BMI}=$ body mass index, PLIN2 = perilipin 2, MC5-R = melanocortin 5 receptor, $S D=$ standard deviation

Levels of PLIN2 and $\mathrm{MC}_{5}$ - $\mathrm{R}$ were compared in the acne group, and no significant difference was found between the sexes ( $p=$ 0.403, $p=0.098$ ). Similarly, in the control group, there was no significant difference in levels of PLIN2 and MC5-R between the sexes ( $p=0.770, p=0.251)$.

According to the severity of acne, differences in PLIN2 and MC5-R levels between female patients (0.104, 0.081, $p>0.05)$ and male patients (0.162, 0.115, $p>0.05)$ were statistically insignificant. However, according to the severity of acne, the differences between PLIN2 and MC5-R levels were statistically significant ( $p<0.05$; Table 2). PLIN2 and MC 5 - $\mathrm{R}$ values were lower in patients with mild acne compared to patients with moderate acne (o.0014, $0.003, p<0.05)$. There was a statistically significant correlation between PLIN2 and MC5-R levels with 79\% accuracy.

Table 2 | Body mass index and perilipin 2 and melanocortin 5 receptor levels by acne severity.

\begin{tabular}{lcccc}
\hline \multirow{2}{*}{ Variable } & \multicolumn{4}{c}{ Acne severity, mean \pm SD } \\
\cline { 2 - 5 } & $\begin{array}{c}\text { Mild } \\
(n=11)\end{array}$ & $\begin{array}{c}\text { Moderate } \\
(n=31)\end{array}$ & $\begin{array}{c}\text { Severe } \\
(\boldsymbol{n}=\mathbf{2 3})\end{array}$ & $\boldsymbol{p}$ \\
\hline $\mathrm{BMI}$ & $21.81 \pm 3.53$ & $21.58 \pm 2.75$ & $22.25 \pm 3.87$ & 0.763 \\
$\mathrm{PLIN2}(\mathrm{ng} / \mathrm{ml})$ & $13.81 \pm 14.59$ & $31.91 \pm 21.59$ & $27.55 \pm 21.69$ & 0.049 \\
$\mathrm{MC} 5-\mathrm{R}(\mathrm{ng} / \mathrm{ml})$ & $7.18 \pm 6.65$ & $17.32 \pm 10.63$ & $14.25 \pm 10.34$ & 0.020 \\
\hline $\mathrm{BMI}=$ body mass index, PLIN2 = perilipin 2, MC5-R = melanocortin 5 receptor, \\
$\mathrm{SD}=$ standard deviation.
\end{tabular}

\section{Discussion}

Although the relationship between acne and sebum secretion has not been clearly identified, the role of sebum secretion in acne development is known. Studies have shown that PLINs play a role in lipid accumulation, which is essential for sebocyte differentiation (6-10). Lipid accumulation decreased in PLIN2-downregulated sebocytes. PLIN2-deficient mouse sebocytes were also determined to be morphologically normal, whereas the size, cell number, and proliferation of sebaceous glands were smaller (8). Increased PLIN2 expression, especially after sebocyte differentiation, suggests that PLIN2 may be a sebocyte differentiation marker $(8,10)$. 
In this study, no difference was found between the acne group and the control group based on PLIN2 levels $(p=0.594)$. However, when acne severity was compared among the acne patients, the group with moderate acne was found to have higher PLIN2 levels compared to the mild acne group $(p=0.014)$. Nevertheless, the same statistical difference between the severe acne group and moderate and mild acne groups was not found $(p=0.110$, $p=0.850$ ).

The study showed no difference in PLIN2 levels between female and male patients, which suggests that PLIN2 levels are not affected by sex. The reason for not detecting a difference in the levels of PLIN2 in the acne and control groups can be attributed to the presence of other factors involved in the pathogenesis of acne, the effect of other LD-related proteins involved in sebocyte differentiation, and the failure to directly assess PLIN2 levels in the skin. A higher level of PLIN2 in the moderate acne group compared to the mild acne group can be interpreted as the role of PLIN2 in acne severity rather than acne pathogenesis. The absence of the same correlation between the severe acne group and moderate and mild acne groups may be interpreted as higher levels of PLIN2 affecting acne severity up to a certain threshold as a result of saturation in sebocyte differentiation. Additional studies with more samples may reveal this relationship more clearly.

A study of MC5-R-deficient mice showed that the MC5-R-deficient group compared to the control group lacked water repulsion and thermoregulation. When the control-group mice's skin lipids were removed, similar effects were observed as seen in $\mathrm{MC}_{5}-\mathrm{R}-$ deficient mice (19). These findings suggest that $\mathrm{MC} 5$ - $\mathrm{R}$ deficiency leads to a decrease in lipid synthesis. The sebaceous glands treated with an MC1-R and MC5-R antagonist, 2,3, diaryl-5 amino- $\{1,2,4\}$ thiadiazole (JNJ-10229570), were found to be small in size and immature (14). Studies have shown that $\mathrm{MC}_{5}-\mathrm{R}$ plays a role in sebocyte differentiation $(12,14,17)$.

This study found no difference between the acne group and the control group based on MC5-R levels $(p=0.213)$. When acne severity was compared among the acne patients, the group with moderate acne was found to have higher $\mathrm{MC}_{5}$ - $\mathrm{R}$ levels compared to the mild acne group ( $p=0.003)$. The same correlation was not detected between the severe acne group and the moderate and mild acne groups ( $p=0.067, p=0.646)$. The study showed no difference in $\mathrm{MC}_{5} \mathrm{R}$ levels between female and male patients, which suggests that $\mathrm{MC}_{5}-\mathrm{R}$ levels are not affected by sex.

With these findings, $\mathrm{MC}_{5}-\mathrm{R}$ levels were not found to be high in the acne group compared to the control group. This study failed to show a difference in $\mathrm{MC}_{5}$ - $\mathrm{R}$ levels between the acne group and the control group. This can be attributed to the multifactorial pathogenesis of acne and the failure to directly assess $\mathrm{MC}_{5}-\mathrm{R}$ levels in the skin.

When acne severity was compared among the acne patients, the group with moderate acne was found to have higher $M_{5}-R$ levels compared to the mild acne group, but MC5-R levels in the severe acne group were not higher than in the moderate and mild acne groups. This finding may be interpreted as higher levels of MC5-R affecting acne severity up to a certain threshold as a result of saturation in sebocyte differentiation or an inadequate sample number.

When the relationship between PLIN2 and $\mathrm{MC}_{5}$ - $\mathrm{R}$ was examined, a statistically significant correlation was found between the two groups. Both PLIN2 and MC5-R play a role in sebocyte differentiation. Therefore, the increase in PLIN2 and MC5-R levels correlates with sebocyte differentiation and is consistent with the literature.

In conclusion, a relationship between acne and PLIN2 and MC5-R levels was not established. A relationship between PLIN2 and $\mathrm{MC}_{5}-\mathrm{R}$ levels and acne severity was also not clearly determined. Nevertheless, this study is preliminary and there are no other studies that examine PLIN2 and $\mathrm{MC}_{5}$-R serum levels in acne patients. PLIN2 and MC5-R levels have only been studied in mouse sebocytes and human sebocyte culture models. Therefore, there is a literature gap. We believe that further studies, especially based on skin samples, are needed to demonstrate the role of PLIN2 and $\mathrm{MC}_{5}$ - $\mathrm{R}$ in the pathogenesis of acne.

\section{References}

1. Degitz K, Placzek M, Borelli C, Plewig G. Pathophysiology of acne. J Dtsch Dermatol Ges. 2007;5:316-23.

2. Gollnick H. From new findings in acne pathogenesis to new approaches in treatment. J Eur Acad Dermatol Venereol. 2015;29:1-7.

3. Zaenglein AL, Pathy AL, Schlosser BJ, Alikhan A, Baldwin HE, Berson DS, et al. Guidelines of care for the management of acne vulgaris. J Am Acad Dermatol. 2016;74:945-73.

4. Thiboutot D. Regulation of human sebaceous glands. J Invest Dermatol. 2004; 123:1-12.

5. Thiboutot D, Gollnick H, Bettoli V, Dréno B, Kang S, Leyden JJ, et al. New insights into the management of acne: an update from the Global Alliance to Improve Outcomes in Acne group. J Am Acad Dermatol. 2009;60:1-50.

6. Sletten A, Seline A, Rudd A, Logsdon M, Listenberger LL. Surface features of the lipid droplet mediate perilipin 2 localization. Biochem Biophys Res Commun. 2014;452:422-7.

7. Khor VK, Shen WJ, Kraemer FB. Lipid droplet metabolism. Curr Opin Clin Nutr Metab Care. 2013;16:632.

8. Dahlhoff M, Camera E, Picardo M, Zouboulis CC, Chan L, Chang BHJ, et al. PLIN2, the major perilipin regulated during sebocyte differentiation, controls sebaceous lipid accumulation in vitro and sebaceous gland size in vivo. Biochim Biophys Acta. 2013;1830:4642-9.

9. Dahlhoff M, Fröhlich T, Arnold GJ, Müller U, Leonhardt H, Zouboulis CC, et al. Characterization of the sebocyte lipid droplet proteome reveals novel potential regulators of sebaceous lipogenesis. Exp Cell Res. 2015;332:146-55.

10. Akimoto N, Sato T, Iwata C, Koshizuka M, Shibata F, Nagai A, et al. Expression of perilipin $A$ on the surface of lipid droplets increases along with the differentiation of hamster sebocytes in vivo and in vitro. J Invest Dermatol. 2005;124:1127-33.

11. Camera E, Dahlhoff M, Ludovici M, Zouboulis CC, Schneider MR. Perilipin 3 modulates specific lipogenic pathways in SZ95 sebocytes. Exp Dermatol. 2014;23: 759-61.

12. Zhang L, Li WH, Anthonavage M, Eisinger M. Melanocortin- 5 receptor: a marker of human sebocyte differentiation. Peptides. 2006;27:413-20.

13. Whang SW, Lee SE, Kim JM, Kim HJ, Jeong SK, Zouboulis CC, et al. Effects of a-melanocyte-stimulating hormone on calcium concentration in $\mathrm{SZ}_{95}$ sebocytes. Exp Dermatol. 2011;20:19-23.

14. Eisinger M, Li WH, Anthonavage M, Pappas A, Zhang L, Rossetti D, et al. A melanocortin receptor 1 and 5 antagonist inhibits sebaceous gland differentiation and the production of sebum-specific lipids. J Dermatol Sci. 2011;63:23-32.

15. Böhm M, Luger TA, Tobin DJ, García-Borrón JC. Melanocortin receptor ligands: new horizons for skin biology and clinical dermatology. J Invest Dermatol. 2006;126:1966-75.

16. Zhang L, Anthonavage M, Huang Q, Li WH, Eisinger M. Proopiomelanocortin peptides and sebogenesis. Ann N Y Acad Sci. 2003;994:154-61.

17. Zhang L, Li WH, Anthonavage M, Pappas A, Rossetti D, Cavender D, et al. Melanocortin-5 receptor and sebogenesis. Eur J Pharmacol. 2011;660:202-6.

18. Thody A, Shuster S. Control of sebaceous gland function in the rat by a-melanocytestimulating hormone. J Endocrinol. 1975;64:503-10.

19. Chen W, Kelly MA, Opitz-Araya X, Thomas RE, Low MJ, Cone RD. Exocrine gland dysfunction in $\mathrm{MC}_{5}$-R-deficient mice: evidence for coordinated regulation of exocrine gland function by melanocortin peptides. Cell. 1997;91:789-98.

20. Doshi A, Zaheer A, Stiller MJ. A comparison of current acne grading systems and proposal of a novel system. Int J Dermatol. 1997;36:416-8. 